\title{
In vitro fertilization and age. When old is too old?
}

\author{
Marek Pokulniewicz ${ }^{1}$, Tadeusz Issat ${ }^{1,2}$, Artur Jakimiuk ${ }^{1,2}$ \\ ${ }^{1}$ Department of Obstetrics, Women's Diseases and Oncogynecology, Central Clinical Hospital of the Ministry of Interior \\ and Administration, Warsaw, Poland \\ ${ }^{2}$ Center for Reproductive Health, Institute of Mother and Child, Warsaw, Poland
}

\begin{abstract}
This case report refers to a 53-year-old female patient who deliberately misinformed the staff of the in vitro fertilization (IVF) clinic using counterfeit documents concerning her real age and underwent the IVF procedure. During the pregnancy it was discovered accidentally that the patient was not telling the whole truth about her real age. In the $34^{\text {th }}$ week of pregnancy two healthy twins have been delivered by a cesarean section. Somehow the trust between the patient and the doctor was compromised, however we can acknowledge this case of postmenopause pregnancy as a therapeutical success for the clinic and for the patient herself. The mother invested her time, emotions and financial resources, and risked her health undergoing IVF procedures and carrying the pregnancy to fulfill her wish and deliver a child. Regardless of her intentions, we can argue about her knowledge concerning this procedure and awareness of threats to pregnancy resulting from her age. The age of childbearing in Western societies has been considerably delayed nowadays, mainly because of social aspects. Many institutions nowadays try to face these aspects, which produces certain medical issues. In this case report authors also present literature review on age and pregnancy after IVF.
\end{abstract}

Key words: IVF, maternal age, donor oocytes, postmenopause.

\section{Introduction}

Infertility affects about $14-20 \%$ of couples nowadays. In modern societies the median maternal age during the first pregnancy is getting higher, mainly for sociological reasons. As these factors accumulate, the age of couples seeking help in assisted reproduction increases.

\section{Case report}

According to the documents, a 43-year-old woman with her partner presented to the infertility clinic in 2005. At the first appointment a standard interview was taken. The patient was pregnant once, in 1980 but terminated the pregnancy by an induced abortion. Her husband already had two children from previous marriage. In 1985, the patient suffered from an intracranial hemorrhage due to an aneurysm rupture and underwent neurosurgical treatment without further neurological defects. In 2001, the woman underwent a gynecological operation - removal of myomas of the uteri. Other operations included removal of varicose veins from thighs. The patient had hypertension, and her medical history also revealed hepatitis $C$ infection.
Before the procedure of the artificial insemination, the couple underwent standard diagnostic procedures, including a karyotype test (normal karyotype in both partners), gynecological ultrasound exam which showed an endometrial polyp, which was further removed hysteroscopically in March 2006 (histopathological exam clinically insignificant) and the partner's sperm analysis (asthenospermia). An internal medicine consultation showed no contraindications to the in vitro fertilization (IVF) procedure. Before the procedure the patient was informed about potential complications and consequences due to pregnancy at an older age.

The transfer (in vitro fertilization-embryo transferintracytoplasmic sperm injection - IVF-ET ICSI) of three donor's oocytes was performed on September 26, 2006. At follow-up visits the patient complained only about occasional diarrhea. In the ultrasound exam on the $24^{\text {th }}$ day of pregnancy, two gestational sacs were discovered - one with a fetus with detectable heartbeat. Later on the patient presented with spotting on the $30^{\text {th }}$ day of pregnancy. The patient was instructed to take medications as previously prescribed - progesterone (vaginally $100 \mathrm{mg} \times 4$ daily), estradiol (orally $3 \times 2 \mathrm{mg}$ daily), drotaverine (orally $3 \times 40 \mathrm{mg}$ daily), combined with well-balanced life style. Throughout this time the patient received methyldopa (orally $3 \times 250 \mathrm{mg}$ daily)

Corresponding author: Marek Pokulniewicz, Department of Obstetrics, Women's Diseases and Oncogynecology, Central Clinical Hospital of the Ministry of Interior and Administration, 137 Wołoska St., 02-507 Warsaw, Poland, e-mail: marek.poko@gmail.com

To cite this article: Pokulniewicz M, Issat T, Jakimiuk A. In vitro fertilization and age. When old is too old? Prz Menopauzalny $2015 ; 14: 71-73$. Submitted: 20.11.2014; Accepted: 27.11.2014. 
and amlodipine (orally $1 \times 5 \mathrm{mg}$ daily) due to her hypertension. The next USG exam, in the $8^{\text {th }}$ week after transfer, revealed properly developing twin dichorionic diamniotic pregnancy, as did the control ultrasound exams in the $13^{\text {th }}, 22^{\text {nd }}$ and $31^{\text {st }}$ weeks of pregnancy. The patient developed a mild anemia, which was effectively treated with iron supplements administered orally.

The first trace of fraud was discovered when the patient was referred to the hospital in the $25^{\text {th }}$ week of pregnancy to receive steroid treatment for threatened preterm delivery. During the admission procedure, a discrepancy between the hospital records and the patient's ID was found. A 45-year-old patient turned out to be 53 in fact. No additional steps were taken besides the entry about the fraud in patient's records. She stayed in the hospital for 4 days during which she underwent a hepatologist consultation (due to her hepatitis C history) with no further recommendations. The ultrasound exam revealed normal, properly developing twin pregnancy. Hospitalization was uneventful and the patient was released home.

The outpatient care followed with no further complications. In the $34^{\text {th }}$ week of pregnancy (on May 13 , 2007), the patient presented to the ward at night with the preterm rapture of the amniotic membrane. Cesarean section, with an epidural anesthesia and standard perioperative i.v. administration of $1 \mathrm{~g}$ cefazolin, was performed, resulting in delivery of two healthy sons $2630 \mathrm{~g} / 48 \mathrm{~cm}, 2500 \mathrm{~g} / 49 \mathrm{~cm}$, with Apgar score 9 in both. Perioperatively $0.4 \mathrm{mg}$ of misoprostol was administered to the patient for prophylaxis of postpartum hemorrhage. The postoperative follow-up was uneventful. On the $7^{\text {th }}$ day following the cesarean section, the patient was discharged from the hospital in good condition with both of her children.

\section{Discussion}

The age of childbearing has been considerably delayed nowadays. Many women deliver their first child at the age of 35 or older. Since the success rate of IVF demonstrates a similar age-related decline as the chance of a natural-pregnancy, women should be informed that the idea of IVF reversing this effect is not correct [1-3].

The Clinical National Institute for Health and Care Excellence (NICE) guidelines for treating people with fertility problems, issued by the Royal College of Obstetricians and Gynaecologists (RCOG) [3], have recently been updated to include a statement [2] in which the authors endorse the recommendation that - if possible - a single embryo transfer should be performed for women aged 39 and under. Although changing the recommendation so that IVF treatment should be available up to the age of 42 provides more choice for women, they should be fully informed about the increased risks associated with pregnancy at advanced age.
Increasing maternal age is a risk factor for almost all pregnancy and perinatal complications. Nevertheless, studies show that while comparing pregnant age groups, there is an increased risk of complications in older woman having IVF (obstetric hemorrhage, preeclampsia, pregnancy induced hypertension, gestational diabetes, higher rate of caesarean section deliveries, unfavorable perinatal outcomes for the neonate - preterm delivery, low birth weight, admission to neonatal intensive care unit). Pre-existing comorbidities are also more common in women with more advanced age, thus also complicating the pregnancy [4-6].

In addition to IVF at older age, there is a larger number of women using oocyte donation. Studies indicate a higher risk of pregnancy induced hypertension (16\% to $40 \%$ of cases, with the greatest risk observed in primiparous women) [4]. At the age over 30, the use of donor eggs was associated with a significantly higher live birth rate compared to the use of woman's own eggs but there was also a downward trend in success rate according to the recipient's age.

When we think about this particular case, a question arises: can we justify such deliberate document frauds? The mother invested her time, emotions and financial resources, and risked her health undergoing IVF procedures and carrying the pregnancy to fulfill her wish and deliver a child. Regardless of her intentions, we can argue about her knowledge concerning this procedure and awareness of threats to pregnancy resulting from her age. In Poland such cases are still rare. Nevertheless, a slightly different case concerning the issue of age in IVF procedures was previously discussed in journals. The case concerned an Italian couple - a 57-year-old woman and a 70 year-old-man - who had decided to undergo artificial heterologous insemination treatment abroad because of restrictions in the Italian law. Having undergone the treatment abroad, the couple had the baby in Italy. The resolution of this issue was difficult because of the conflict between the parents' right to self-determination and unborn child's protection. Judges' opinion was that those parents were too old and unfit to be parents [7]. In the final decision the judge ordered to deprive parents of their 1-year-old child and subsequently give it up for adoption. No legal case was brought against the couple.

In the end, we have to remember that despite the ethical side of this case, conceiving healthy twins in this age can be seen as a great success. The success rate of IVF demonstrates a similar age-related decline as the chance of a natural-pregnancy. Even for a woman in the age that she claimed to be, the chances of a successful procedure which was followed by mostly uncomplicated twin pregnancy would be quite fortunate. Recent registry data suggest that donor oocyte recipients have stable rates of pregnancy outcomes before the age of 45 , after which there is a small but steady and signifi- 
cant decline [8]. A better compliance might be endorsed with better education and information, ensuring that women seek help earlier and reconcile their need of having a child with better outcomes for children and their own selves.

\section{Disclosure}

Authors report no conflict of interest.

\section{References}

1. http://www.rcog.org.uk/what-we-do/campaigning-and-opinions/statement/rcog-statement-later-maternal-age.

2. http://www.rcog.org.uk/what-we-do/campaigning-and-opinions/statement/rcog-statement-updated-nice-guidance-treating-people-f.
3. NICE Guideline. Assessment and treatment for people with fertility problems. 11.2 Female Age; http://www.ncbi.nlm.nih.gov/books/ NBK45935/pdf/TOC.pdf.

4. In Vitro Fertilisation: Perinatal Risks and Early Childhood Outcomes. 5.1 Maternal Age; http://www.rcog.org.uk/files/rcog-corp/060612SAC8.pdf.

5. Reddy UM, Wapner RJ, Rebar RW, Tasca RJ. Infertility, assisted reproductive technology, and adverse pregnancy outcomes: executive summary of a National Institute of Child Health and Human Development workshop. Obstet Gynecol 2007; 109: 967-977.

6. Healy DL, Breheny S, Halliday J, et al. Prevalence and risk factors for obstetric haemorrhage in 6730 singleton births after assisted reproductive technology in Victoria Australia. Hum Reprod 2010; 25: 265-274.

7. Gulino M, Pacchiarotti A, Vergallo GM, Frati P. Is age the limit for human-assisted reproduction techniques? 'Yes', said an Italian judge. J Med Ethics 2013; 39: 250-252.

8. Yeh JS, Steward RG, Dude AM, et al. Pregnancy outcomes decline in recipients over age 44: an analysis of 27,959 fresh donor oocyte in vitro fertilization cycles from the Society for Assisted Reproductive Technology. Fertil Steril 2014; 101: 1331-1336. 